\title{
Principals' Value Leadership and Campus Football Development: Ideal Pursuits and Present Dilemmas
}

\author{
Haoyang Zhuge* \\ Graduate Education College, Shandong Sports University, Jinan, 250100, China \\ "Corresponding Author's Email: 736207191@qq.com
}

\begin{abstract}
Adopting the literature research methodology and field survey method, this thesis takes the new era of school soccer as the background, and the combination of value leadership of principals and school soccer development as the problemoriented point. The value leadership of principals in campus soccer development is constructed from three dimensions: cognition, transmission and internalization; integration and guidance; implementation and guarantee. Through the inspection and research of schools featuring campus soccer and the combing of relevant literature, the present dilemmas of principals' value leadership are as follows: cognitive biases deviation leads to the solidification of medals mania and formalism; insufficient cultural construction of in value hinders the transmission of soccer spirit; setbacks in value guidance and integration restrict talent cultivation. Last but not least, based on the existing problems, this thesis has proposed the countermeasures including internal and external training, multi-party coordination and top-level design.
\end{abstract}

Keywords: school soccer, principal, value leadership, ideal pursuits, present dilemmas

\section{INTRODUCTION}

Since school soccer was led by the education department in 2015, its development in China has been flourishing, achieving breakthroughs in school soccer education and teaching system, league organization as well as teachers' training in the context of integration of sports and education. However, due to the deep-rooted traditional education ideology in China, there are still differences in the perception of the value of campus soccer among all sectors of society. Besides, the culture and value of campus soccer is neglected resulting from pursuing numerical results and immediate successes. In addition, the public's perception of its value remains at the low level featuring technical and tactical teaching, competition and elite talent training, which deviates from the core value concept focusing on nurturing trainees. Therefore, it is necessary for campus soccer to explore a connotative development path from a new standpoint. As the backbone of campus soccer, basing on how to improve students' physical health and tactical skills, principals of these schools are supposed to set promoting students' sound personality as the core, and enhancing the soft power of China's soccer culture as the visionary goal as well as leverage their leadership to form the correct value orientation of campus soccer inside and outside the campus, which has been an important issue for its development at a new stage.

Based on the above issue, this thesis aims to analyze the philosophical logic of principals' value leadership in leading the development of campus soccer from the perspective of principals' value leadership. By literature review, it's found that at the beginning of this century, Hong Kong scholar Zheng Yanxiang proposed five vectors of leadership for educational organization leaders to exert leadership, which are structural leadership, interpersonal leadership, political leadership, educational and pedagogical leadership together with cultural leadership. [1] Based on the connotation and extension of cultural leadership and guided by the core value system, modern scholars have proposed the new term "principals' value leadership". Research on principals' value leadership has focused on three aspects: conceptual connotation, classification structure, and leadership enhancement in school contexts. In terms of conceptual connotation, scholars consider principal's value leadership as the principal's ability to lead, regulate, integrate, transform, and practice the values of teachers, students, and socially relevant interest groups in order to achieve school development goals, [2] as well as the 
ability to understand, apply, unify, create, and practice a core value. [3] Other scholars, from the perspective of leadership purpose, consider principals' value leadership as the ability to use the art of value leadership to solve problems in school management practices, regulate the behavior of educational organizations and members, and achieve organizational goals. [4] In terms of classification structure, scholars mainly classify it into abilities of value identification and judgment, value guidance, value defense and expression ability, value integration as well as value practice [5] In terms of leadership enhancement, relevant studies have pointed out that the current school culture value development is characterized by value deficiency, value uncertainty, value distortion, value hypocrisy, value isolation, "authoritative management", "rigid management", and "achievement first". Based on this dilemma, the study proposes that principals should refine their values by clarifying the school's core values, transforming values, reflecting the core values in their own behavior and interpersonal interactions, and externalizing them to school organization. What's more, principals should strengthen value leadership to influence the awareness and behavior of internal and external school personnel as well as promote value-enhancing solutions.[6]

In summary, the current academic research on principals' leadership and principals' value leadership has become systematic, providing a solid theoretical foundation for this study. However, a mature theoretical combination of leadership theory and school soccer development has not yet been formed. In view of this, this thesis, with relevant literature research, by visiting 28 representative schools featuring campus soccer in the districts and counties of Jinan and integrating the connotative concepts related to value leadership, attempts to construct various dimensions of principals' value leadership on campus soccer development, summarize and discern the ideal orientations as well as actual problems of each dimension, and propose countermeasures to solve the problems in a objective and dialectical manner.

\section{THE IDEAL PURSUITS OF VALUE LEADERSHIP IN SCHOOL SOCCER DEVELOPMENT BY PRINCIPALS}

"Value" is the satisfaction of the subject's need for the object, and the subject is pluralistic and mutually transformative. Every organization has multiple subjects with different values. When it comes to schools, which are "loose organizations" with different roles, school leaders need to play a leadership role in the mainstream values to establish a strong value culture of school soccer [7] Value leadership of school soccer development by the principal means that the principal injects correct school soccer values into the target of leadership and forms a value community, so that the relevant multiple subjects can play a joint role to achieve the goals of school soccer. From the perspective of open system, the target of value leadership of school soccer by principals is not only the teachers and students inside the school, but also the government departments, social organizations, communities, parents and other system elements outside the school.

Combining scholars' research on value leadership and the development needs of campus soccer cause, the author divides the value leadership of school soccer development by principals into: principals' leadership in cognition, transmission and internalization of campus soccer values; leadership in guidance and integration of campus soccer values; and leadership in implementation and guarantee of campus soccer values.

\subsection{Cognition, transmission and internalization of campus soccer values under the leadership of principals}

Since the 18th National Congress of the Party, China has elevated "educating people with moral character" to the fundamental task of socialist modern education. The policy of campus soccer development has made it the core goal to nurture talents for the country and the Party, and to cultivate builders and successors of the socialist cause with collectivism and patriotism. Especially since school soccer is led by the education system, it centers more on quality education value to improve personality, refine will and enhance collective cohesion. Through guiding students to participate in school soccer activities, it not only creates favorable conditions for improving their physical health, but also provides an effective vehicle for improving their IQ, emotional intelligence and social adaptability. With the deepening of school sports reforms, students' physical fitness conditions have improved significantly, yet school leaders and teachers lack a deep enough understanding of the value of quality education in school sports and have superficial ideological perceptions.

In the process of leading the development of school soccer, principals with excellent value leadership can not only truly recognize the core role of school soccer in "sound personality" and "building moral character" as well as establish the value of quality education, but also lead soccer teachers to impart moral qualities and abilities to students in a deeper way, so that they can be transformed into students' own internal ideology and external behavioral norms. For example, the principal of Dajin Elementary School in Huaiyin District in Jinan conveyed the nurturing value of school soccer to trainers by organizing job training so that they could guide their players to establish the behavioral qualities of respecting opponents and referees during matches. After the matches, they would organize players to express their gratitude to coaches and players against them, as wel as those field staff. There are mainly two reasons: firstly, for the pressure and challenges brought by opponents which in 
turn train themselves. Secondly, for the staff's hard work and provision of a good playing environment. Through moral externalization and behavioral presentation, teachers, students and parents watching the game can also be inculcated with "moral empathy" and improve their own moral quality. In addition, when it comes to a close match, the process of practicing tactical cooperation in soccer class serves as a good opportunity for students to develop a sense of shouldering responsibility and promoting cooperation, which are the qualities that students should harbor when they integrate into society after graduation.

It can be said that school soccer is a microcosm of social life, or a very intuitive moral education campaign. Its effect on education is much better than theoretical indoctrination. Therefore, the recognition, transmission and internalization of the comprehensive nurturing value of campus soccer under the leadership of the principal is the key to practicing original missions of campus soccer development.

\subsection{Guidance and integration of campus soccer values under the leadership of principals}

In a campus where different value subjects converge, principals should think about how to grasp the subjective value perceptions related to the interests of campus soccer, inject the core cultural values of campus soccer into the members, integrate them to form a value consensus and avoid conflicts arising from value differences.

Students are the main participants of campus soccer and school leaders, staff and students' parents are the main stakeholders. If the value of a certain subject wants the recognition of groups inside and outside the organization, there must be a deep and strong cultural system behind it. Therefore, the spiritual and cultural development of campus soccer largely influences the generation of students' interest in soccer and the formation of stakeholders' concepts of soccer value. Principals should find a core soccer spiritual culture that deeply guides and integrates the soccer values of all stakeholders on campus. For example, in a previous training session for principals, Jiang Xiushan, the principal of Jiaozhou Zhongyun Zhenhua Education Group, used the school soccer business as an incubator for school development, guiding teachers to establish a "student-centered" soccer teaching philosophy that respects interests and guides students to build up qualities of "tenacity, unity and cooperation, never give up" and "if there is soccer in your heart, the world is a soccer field". Driven by such spiritual culture, the school soccer career has developed rapidly, leading to leaps and bounds in other areas of work, which has been widely recognized by students' parents and the community.

\subsection{Implementation and guarantee of campus soccer values under the leadership of principals}

Whether the core values of campus soccer can play its role to the most depends to a large extent on the principal's leadership in the practical actions of the school. Specifically, the principal's ability to explore breakthroughs in the practice of campus soccer values from the artifact level, behavior level to the institutional level will be the key to ensuring the function of campus soccer values.

First, as far as the artifact level is concerned, principals should lead their schools to create a positive soccer culture by planning soccer facilities, setting up bulletin boards, and collecting soccer books and video materials. For example, the principal of Futai Elementary School in Zhangqiu District in Jinan, led the school to build a campus soccer theme corridor and theme park, create a campus soccer documentary and a soccer literature series, as well as design a mascot with soccer elements.

Second, on the behavioral level, principals should guide their schools to organize regular leagues and cultural festivals with symbolic soccer culture. For example, Minsheng Street Elementary School in Jinan has established soccer interest groups, clubs and societies, set up an "Open Day for Parents of Campus Football" for students to play soccer matches with their parents, honored advanced figures in campus soccer through a ceremony, and established a campus soccer information platform to showcase the special achievements of campus soccer.

Finally, at the institutional level, the system is a powerful guarantee for realizing campus soccer values. To break the obstacles to the value of campus soccer leadership, it is not enough to solely rely on the spiritual and cultural level of propaganda and leadership, but also requires dependence on instrumental and rational institutional norms. First, the principal should lead the school to develop a propaganda system for soccer culture and stipulate hard indicators for cultural construction. Second, they should establish mature systems of teaching, training and competition, sports protection and security, teacher training, reward and punishment, as well as school training coordination.

\section{PRESENT DILEMMAS OF PRINCIPALS' VALUE LEADERSHIP OF SCHOOL SOCCER DEVELOPMENT}

\subsection{Value perception bias leads to the solidification of medals mania and formalism}

Under the influence of the traditional education model that emphasizes intellectual education over moral education and marks over quality, school principals, 
teachers, parents and related groups still singularly regard school sports as "limb education". Principals lack the cognitive understanding and connotative guidance of the value function of school soccer. The value perception bias inevitably causes purposeful deviation of leadership behavior. The development of school soccer has both achievements and contradictions. Inevitably, there are unhealthy practices of medal mania and formalism at the school level. In a field study of some districts and counties in Jinan including Jiyang District, Southern mountains District, and Pingyin County, where school soccer developed late, the author found that some schools with school soccer characteristics focused only on immediate interest as well as formal indicators in order to meet the target tasks assigned by the higher levels, and the principals' perception and implementation of the core goals of school soccer were not in place. Instead of focusing school soccer on nurturing people, they narrowed the goal to improve the competitiveness and athletic performance of the team, and regarded winning medals as a way to enhance political performance. This has led to a gradual weakening of the overall nurturing effect of school soccer as a whole, and ordinary schools have become "concentration camps" for the development of elite athletes. In addition, some special schools want to keep the brand of "school featuring soccer", but also to ensure the rate of advancement. Therefore, the contradiction of various interests impedes the advance of goals. There even emerges the phenomenon of "features only for leaders' inspection ".

The fundamental problem that restricts the development of school soccer is that the stakeholder groups have not formed a consensus on the value of school soccer's moral education function. Especially for schools featuring soccer, if the principal himself does not correctly recognize the value of school soccer, how can he talk about guiding school members and social pluralists to form positive value judgments?

\subsection{Insufficient cultural construction hinders the transmission of soccer spirit}

At present, principals hold ambiguous, single, biased and even indifferent attitudes in leading the construction of campus soccer culture that is in its exploration stage. First of all, ambiguous cognition of campus soccer spirit culture and single transmission method. In the process of fieldwork, the author found that a considerable number of schools had built a publicity board with soccer slogans and a display board for the honor of the school team in a prominent position on campus, but there was no other carrier for the transmission of soccer spiritual culture. In addition, in terms of what is the spiritual culture of school soccer, some principals' perceptions are rather vague, only providing answers of "fighting", "winning" and "championship spirit" from the perspective of competition. Secondly, the culture of immediate success and profit has resulted in perverse values for students. For example, some schools indeed carry out activities related to soccer handwriting, soccer essays and photography exhibitions, but they fail to persist in the long run, leaving students to complete their works in a short period of time when there are inspections of their superiors, and some even organize teachers to do so. This not only exerts pressure on teachers and students, but also tends to make students form a value bias of " muddling through", causing counterproductive effects of spiritual culture transmission.

\subsection{Setbacks in value guidance and integration restrict the talent training channel}

Through the research, the author found that the biggest constraints to the cultivation of school soccer talents in Jinan are the "contradiction between learning and training", in other words, students' academic studies and soccer training, parents' psychological anxiety about students' sports safety, and the integration gap in the cultivation channel of school soccer talents. During the communication process with the principals of Xiaojin Primary School in Huaiyin District, Meili Lake Primary School, Huashan Primary School in Licheng District, the fourth middle school in Laiwu District as well as the staff of local education and sports departments, the author has learned that some children in the junior high school soccer team were forced to shorten training time after entering high school due to the pressure of college entrance exams. Besides. parents and teachers expected students to give up soccer training in that any injury will cause delay in courses. Students who play soccer well in elementary schools who enter junior high school fail to continue their interest in soccer and set aside soccer training after entering junior high schools under the zoning enrollment policy. Because their junior high schools don't feature soccer and lack soccer teams. In addition, Jinan Cascade School and other schools implement the "enrollment lottery" policy at the compulsory education level, and lack enrollment policies for soccer students with special talents, so the school team players can only be selected from class and grade leagues.

The value cultivation of campus soccer talents is necessary for soccer reserves in China, but in the macro context of increasingly competitive society and accelerating pace of life, there are inevitably various unfavorable factors that restrict the value. At present, the fence of school soccer value leadership does not lie in the value bias of members within the school, but the key lies in the "score anxiety", "promotion anxiety" and "safety anxiety" of parents and society towards students. The ideology of score and diploma-based education is deeply rooted, and many people believe that allowing students to participate in soccer activities will affect their academic performance and hinder their future path to higher education, in addition to possibly causing unknown 
physical injuries. This contradiction between school training and sports safety issues has caused parents to hold an opposing attitude toward the development of campus soccer activities, severely hindering students' freedom to participate in soccer activities and depriving them of the right to engage in physical exercise. In this context, the guidance and integration of the core values of school soccer by principals is bound to be hindered. Coupled with the lack of policies for the advancement of soccer students, the prejudice of parents and society toward school soccer will become more serious, restricting the smooth development of school soccer talent.

\section{RESPONSES}

\subsection{Internal and external training to enhance the cognition, transmission and internalization of the value of school soccer for principals' leadership}

Learning relevant theoretical knowledge. Principals should strengthen the study of educational leadership theory, school sports theory, campus soccer theory and relevant policy documents, deeply understand the national development orientation of campus soccer and the nurturing value of campus soccer, as well as lead members to achieve the development goals of campus soccer in schools. [8]

Explore the publicity channels of the cultural value of campus soccer. First, strengthen public opinion leadership through various informational media or social platforms to report campus football-related activities, vigorously promote the significance of carrying out campus soccer activities, create a healthy campus soccer cultural atmosphere inside and outside the school, and promote the spiritual quality of campus soccer. Secondly, organize school soccer cultural festivals in a regular manner, integrate elements of art, labor, general studies and other disciplines, invite parents and social groups to participate at appropriate times, and set up "school soccer open days".

\subsection{Multi-party coordination to promote principals' ability to lead and integrate the value of campus soccer}

Establish a campus soccer support alliance. Principals should accurately identify the value orientation of school members, parents, and social groups towards the cause of campus soccer, and identify supporters, neutrals, and opponents. For the opponents, they need guidance to change their value bias towards school soccer through effective negotiation. The supporters and neutrals need to be motivated to take initiative and creativity through certain incentives, and to be willingly submissive to the organization's leadership through personal leadership and by listening to and caring for school members, thus building a "support coalition" for school soccer. Specific measures can be divided into the following points.

First, establish a cooperative alliance between families, schools and communities, with school soccer as the fulcrum. Parents can learn more about the real value of school soccer through parent-child activities and special exchanges by setting up "school soccer open days" which bring parents to campus. Parents are invited to participate in the decision-making process of school soccer, and their opinions and suggestions are sought to make the management of school soccer more scientific and innovative. At the end of the semester, parents are awarded the title of "Outstanding Parent in School Football".

Second, cooperate with professional soccer clubs in the region to make up for the shortage of school soccer teachers, field equipment and teaching resources through communication, talent introduction and purchase of services, as well as learn advanced teaching concepts.

Third, communicate with head teachers and other subject teachers to understand their attitudes toward campus soccer, convey to them the correct concept of campus soccer values, and guide teachers of various subjects to integrate soccer elements into the subjects they teach.

\subsection{Strengthen top-level design to ensure the implementation and guaranteeing ability of principals to lead campus soccer values}

First, build a policy system for campus soccer in terms of coordination of school training, articulation to higher education, and sports accident insurance in order to extend the policy to every school.

Second, establish an assessment system for the cultural construction of schools with campus soccer characteristics. Assess the level of cultural construction of campus soccer in four aspects: material culture, spiritual culture, institutional culture, and behavioral culture. [9]

Third, organize regular training courses for school principals of schools featuring soccer to optimize the training content of the campus soccer leadership module for principals based on the macro policy of national campus soccer, and to guide principals to establish the correct value concept of campus soccer.

\section{CONCLUSION}

The value of school soccer, with school as the main field, is to enable students to develop a strong interest in soccer and master basic soccer skills through soccer activities. In addition, it aims to play the school soccer's role in quality education for all students, and to cultivate 
a sound personality of students.[10] In turn, on the basis of popularizing soccer, high level soccer talents can be explored, and the whole process of "nurturing the heart", "nurturing the intellect", "nurturing the body" and "nurturing the talent" can be carried out.

The key for principals to use the art of value leadership to crack the problem of school soccer development still lies in the construction of educational philosophy and environment. However, it is difficult to solve the current general dilemma by relying only on the leadership role played by the principal as a unilateral subject. Schools, society and the government must form an organic multi-governance system, pay attention to the value leadership of the principal's campus soccer development from multiple dimensions, effectively leverage the core origin of campus soccer education, set the establishment of moral education as their fundamental goal, and truly reflect the greatest value of campus soccer as a national policy of education.

\section{REFERENCES}

[1] Zheng Yanxiang. New Paradigm of Educational Leadership and Reform [M]. Shanghai Education Press:Shanghai,2005.9:197.

[2] Yao Xiaolan. The training strategies of rural principals from the perspective of value leadership [J]. Journal of Jilin Provincial College of Education,2020,36(10):75-78.

[3] Li Zhengtao. How can principals achieve value-based leadership? [J]. Primary and Secondary School Management,2011(01):5-8.

[4] Chen Guoan. Value-based leadership of principals [J]. People's Education,2012(10):17-19.

[5] Shi C-Y. Talking about the value leadership of principals[J]. Primary and Secondary School Management, 2007(07):4-6.

[6] Wang Shuifa. The logic of school development based on the perspective of principal's value leadership[J]. Education Research,2012,33(09):49-52.

[7] Liu Yanru. Value-based leadership [D]. Shanghai:East China Normal University.2019(09)

[8] yao jian. Research on the implementation of campus football promotion led by principals [J]. Journal of Beijing sport university,2017,40(04):75-82.

[9] Lu Xiongwen. Dictionary of Management [M]. Shanghai: Shanghai Dictionary Publishing House, 2013.12:49-51.

[10] Zhang Xingquan. New interpretation and construction strategy of campus football culture from the perspective of cultural characteristics theory $[\mathrm{J}]$. Journal of shenyang physical education university,2018,37(04):59-63+104. 